\title{
Distribuição e caracterização de diferentes populações de Triatoma brasiliensis Neiva, 1911 (Hemiptera, Reduviidae, Triatominae)
}

\author{
Distribution and characterization of different \\ populations of Triatoma brasiliensis Neiva, 1911 \\ (Hemiptera, Reduviidae, Triatominae)
}

\footnotetext{
1 Núcleo de Informatização/ Coleção Entomológica

Departamento de Entomologia, Instituto Oswaldo Cruz, Fundação Oswaldo Cruz. Av. Brasil 4365, C.P. 926, Rio de Janeiro, $R J$ 21045-900, Brasil. jcosta@ioc.fiocruz.br
}

\begin{abstract}
Triatoma brasiliensis Neiva, 1911 is now considered the most important Chagas disease vector in the semiarid zones of northeastern Brazil. Four distinct populations of T. brasiliensis have been identified by multidisciplinary studies: brasiliensis (Caicó, RN), melânica (Espinosa, MG), macromelasoma (Petrolina, PE), and juazeiro (Juazeiro, BA). By scanning electron microscopy of egg exochorion, each population displayed a distinct ornamentation pattern. The brasiliensis, macromelasoma, and juazeiro populations were found in both artificial ecotopes and the wild, while the melânica population was collected only in the wild. Isoenzymatic analysis detected phenotypic and genetic differences among them, with the melânica population being the most distinct. The brasiliensis population is the most important one from an epidemiological point of view, with the widest geographic distribution, the highest Trypanosoma cruzi infection rate, and occupying a wide variety of ecotopes.
\end{abstract}

Key words Chagas Disease; Triatominae; Triatoma brasiliensis; Insect Vectors

Resumo Triatoma brasiliensis Neiva, 1911 é agora considerado o mais importante vetor da doença de Chagas nas áreas semi-áridas do nordeste do Brasil. Quatro populações distintas de $\mathrm{T}$. brasiliensis foram evidenciadas através de estudos multidisciplinares: brasiliensis (Caicó, RN), melânica (Espinosa, MG), macromelasoma (Petrolina, PE) e juazeiro (Juazeiro, BA). A microscopia eletrônica de varredura do exocório dos ovos de cada população evidenciou um padrão de ornamentação distinto para cada uma delas. As populações brasiliensis, macromelasoma, $e$ juazeiro foram encontradas em ecótopos artificiais e naturais enquanto a população melânica foi localizada apenas em ecótopos naturais. As análises isoenzimáticas detectaram diferenças fenotípicas entre todas as populações, sendo a melânica a mais distinta. Sob o ponto de vista epidemiológico, a população brasiliensis é a principal, por apresentar a mais ampla distribuição geográfica, o mais alto índice de infecção natural por Trypanosoma cruzi e por ocupar ampla variedade de ecótopos.

Palavras-chave Doença de Chagas; Triatominae; Triatoma brasiliensis; Insetos Vetores 


\section{Introdução}

Triatoma brasiliensis foi descrito, em 1911, por Neiva, que examinou espécimes provenientes de Caicó, Rio Grande do Norte. Essa espécie, que era considerada de importância secundária no que diz respeito à capacidade vetorial $\mathrm{e}$ às medidas de controle, tornou-se, atualmente, uma das prioridades dos órgãos executivos do Ministério da Saúde, por ser considerada o principal vetor da doença de Chagas nas zonas semi-áridas do Nordeste do Brasil.

T. brasiliensis apresenta ampla distribuição geográfica, tendo sido registrada em vários estados: Alagoas, Bahia, Ceará, Maranhão, Minas Gerais, Paraíba, Pernambuco, Piauí, Rio Grande do Norte, Sergipe e Tocantins (Silveira et al., 1984).

Essa espécie apresenta variações cromáticas no pronoto, patas e asas, que levaram a descrição de subespécies. Neiva \& Lent (1941) descreveram T. brasiliensis melanica, baseando-se em espécimes coletados em Espinosa, Minas Gerais. Galvão (1956) descreveu um novo padrão, discriminando-o como T. brasiliensis macromelasoma, a partir da análise de espécimes coletados em Juazeiro, na Bahia, e Petrolina, em Pernambuco, designando Petrolina como localidade-tipo. Nesse estudo, o autor, atribuindo nomenclatura trinomial para o primeiro padrão descrito, elabora uma chave taxonômica para as três subespécies de T. brasiliensis. As subespécies foram descritas com base nos caracteres cromáticos do protórax, das patas e asas. Lent \& Wygodzinsky (1979) consideraram-nas em sinonímia, justificando que padrões intermediários podem ser encontrados.

Com o intuito de ampliar os conhecimentos sobre a variabilidade existente entre quatro distintas populações de T. brasiliensis, foram realizados estudos multidisciplinares:

1) morfológicos - análise das estruturas genitais externas dos machos, microscopia eletrônica de varredura e morfometria dos ovos (Costa et al., 1997a);

2) biológicos - determinação dos períodos de intermuda e de resistência ao jejum (Costa \& Marchon-Silva, 1998), caracterização dos ecótopos, do percentual de infecção natural e das possíveis fontes alimentares através do teste de precipitina (Costa et al., 1998); e realização de cruzamentos recíprocos entre os diferentes padrões cromáticos (Costa et al., 1996);

3) isoenzimáticos - análise de nove loci gênicos (Costa et al., 1997b).

Como base para este estudo, foram utilizados insetos depositados nas coleções Herman Lent e Cesar Pinto, do Instituto Oswaldo Cruz, exemplares do insetário do Laboratório Nacional e Internacional de Referência em Taxonomia de Triatomíneos e também espécimes coletados no campo, nas localidades onde diferentes padrões cromáticos foram descritos: Caicó, no Rio Grande do Norte (padrão brasiliensis), Espinosa, em Minas Gerais (padrão melânica), Petrolina, em Pernambuco (padrão macromelasoma) e Juazeiro, na Bahia (padrão juazeiro). Na Bahia, que apresenta fronteiras com nove estados, foram realizadas coletas em mais quatro localidades. Os espécimes capturados deram origem a colônias que estão sendo mantidas no laboratório da Coleção Entomológica há quatro anos. Foram observados padrões cromáticos estáveis, homogêneos e característicos, para cada uma dessas populações, sugerindo que essas diferenças fenotípicas ocorrem em virtude de componentes genéticos. A análise comparativa das estruturas genitais externas mostrou variações intrapopulacionais, dentro de um mesmo padrão cromático e ausência de características interpopulacionais, que impediram diferenciar as quatro populações. O exocório e o opérculo dos ovos evidenciaram padrões morfológicos distintos analisados através da microscopia eletrônica de varredura; a morfometria também apresentou resultados estatisticamente significativos, na distinção dessas populações, apesar da superposição de valores observada. Na bionomia, tanto para os períodos de intermuda, quanto para a resistência ao jejum, foram demonstradas diferenças estatisticamente significativas entre as populações. Nas coletas realizadas, as populações brasiliensis, macromelasoma e juazeiro foram observadas tanto em ambientes naturais quanto em artificiais, enquanto a população melânica foi registrada apenas nos ecótopos silvestres. Nos percentuais de infecção natural por Trypanosoma cruzi, 15\% dos espécimes da população brasiliensis e $6,6 \%$ dos de melânica foram encontrados infectados. Não tendo sido detectada infecção natural nos espécimes das populações macromelasoma e juazeiro. Com relação às fontes alimentares, foi observado ecletismo alimentar para as quatro populações. Os estudos isoenzimáticos revelaram que as populações possuem características genéticas peculiares, sendo a melânica a mais distinta. Os cruzamentos experimentais recíprocos entre os diferentes padrões cromáticos encontram-se em andamento, mas para $\mathrm{F}_{1}$ foi demonstrada compatibilidade genética entre todos os padrões cromáticos.

Do ponto de vista epidemiológico, o padrão cromático brasiliensis é o mais importante, por diversas razões: 1) apresenta a mais ampla dis- 
tribuição geográfica (comparando-se exemplares da coleção Herman Lent, esse padrão cromático ocorre no Ceará, Paraíba, Piauí e Rio Grande do Norte); 2) tem o mais alto percentual de infecção natural; 3) foi coletado no ambiente silvestre, no peri e no intradomicílio; 4)através do teste de precipitina, foi evidenciado ecletismo alimentar.

Pelos estudos realizados, $T$. brasiliensis apresentou-se como uma espécie politípica, constituída por diferentes populações alopátricas com potenciais epidemiológicos distintos.

\section{Agradecimentos}

Agradecemos à Fundação Nacional de Saúde e ao Conselho Nacional de Desenvolvimento Científico e Tecnológico pelo apoio na realização do trabalho.

\section{Referências}

COSTA, J.; ALMEIDA, J. R.; DUARTE, R.; MARCHONSILVA, V. \& PACHECO, R. S., 1998. Ecotopes, natural infection and trophic resouces of Triatoma brasiliensis (Hemiptera, Reduviidae, Triatominae). Memórias do Instituto Oswaldo Cruz, 93:7-13.

COSTA, J.; BARTH, O. M.; MARCHON-SILVA, V.; ALMEIDA, C. E.; FREITAS-SIBAJEV, M. G. R. \& PANZERA, F., 1997a. Morphological studies on the Triatoma brasiliensis Neiva, 1911 (Hemiptera, Reduviidae, Triatominae). Genital structures and eggs of different chromatic forms. Memórias do Instituto Oswaldo Cruz, 92:493-498.

COSTA, J.; FREITAS-SIBAJEV, M. G. R.; MARCHONSILVA, V.; PIRES, M. Q. \& PACHECO, R. S., 1997b. Isoenzymes detect variation in populations of Triatoma brasiliensis (Hemiptera: Reduviidae: Triatominae). Memórias do Instituto Oswaldo Cruz, 92:459-464.

COSTA, J. \& MARCHON-SILVA, V., 1998. Período de intermuda e resistência ao jejum de diferentes populações de Triatoma brasiliensis Neiva, 1911 (Hemiptera, Reduviidae, Triatominae). Entomología y Vectores, 5:23-34.
COSTA, J. M.; MARCHON-SILVA, V. \& ALMEIDA, J. R., 1996. Does hybridization clarify the taxonomic status of different melanic forms of Triatoma brasiliensis Neiva, 1911 (Hemiptera, Reduviidae, Triatominae). Memórias do Instituto Oswaldo Cruz, 91:128.

GALVÃO, A. B., 1956. Triatoma brasiliensis macromelasoma $\mathrm{n}$. subsp. (Reduviidae, Hemiptera). Revista Brasileira de Malariologia e Doenças Tropicais, 7:455-457.

LENT, H. \& WYGODZINSKY, P., 1979. Revision of the Triatominae (Hemiptera, Reduviidae), and their significance as vectors of Chagas' disease. Bulletin of the American Museum of Natural History, 163:123-520.

NEIVA, A. \& LENT, H., 1941. Sinopse dos Triatomíneos. Revista de Entomologia, 12:62-92.

SILVEIRA, A. C.; FEITOSA, V. R. \& BORGES, R., 1984. Distribuição de triatomíneos capturados no ambiente domiciliar, no período 1975/83, Brasil. Revista Brasileira de Malariologia e Doenças Tropicais, 36:15-312. 